\title{
EXPECT THE UNEXPECTED: PROCESSING OF SENSORY INFORMATION IN THE ANTICIPATION OF PREDICTABLE AND UNPREDICTABLE THREATS
}

\section{Eva Koderman}

University of Primorska, Slovenia

E-mail: koderman.eva@gmail.com

\begin{abstract}
Anxiety is characterized by a sustained state of heightened vigilance due to uncertain danger, producing increased attention to a perceived threat in one's environment. To further examine this exploited the temporal resolution afforded by event-related potentials to investigate the impact of predictability of threat on early perceptual activity. We recruited 28 participants and utilized a within-subject design to examine hypervigilance in anticipation of shock, unpleasant picture and unpleasant sound during a task with unpredictable, predictable and no threat. We investigated if habituation to stimuli was present by asking the participants to rate unpleasantness and intensity of the stimuli before and after the experiment. We observed hypervigilance in the unpredictable threat of shock. Habituation was observed for the visual stimuli. The present study suggests that unpredictability enhances attentional engagement with neutral somatosensory stimuli when the threat is of the same modality, meaning we observed the presence of hypervigilance which is a characteristic of anxiety.
\end{abstract}

Key-words: sensory processing, anxiety, unpredictable threats, EEG, N1

\section{Introduction}

In recent years we have witnessed a proliferation of studies focusing on sensory processing of threats in hope to better understand the underlying mechanisms of anxiety when an individual goes into a state of hypervigilance (Baas et al. 2006; Cornwell et al. 2007; Driver and Noesselt 2008; Dunning, DelDonno and Hajcak 2013; Sharvit et al. 2016; Cecchetti et al. 2016; Dieterich, Endrass and Kathmann 2016; Domínguez-Borràs et al. 2017). It is still unclear how the information in this state is processed, specifically how different sensory modalities interact among themselves. Unwanted possibilites or threats from the environment can turn out to be predictable or unpredictable and how we react to them can be critical for our survival. 
Unpredictable threats induce sustained anxiety, followed by vigilance, whereas imminent, predictble threat leads to phasic fear with selective attention (Grillon et al. 2004). Recent researches suggest that threatening context can sensitize reactivity to stimuli in all sensory modalities (Baas et al. 2006; Cornwell et al. 2007; Dunning, DelDonno and Hajcak 2013; Sharvit et al. 2016), however it remains unclear whether these neural mechanisms occur across different sensory modalities crossmodally, supramodally or intramodally (Driver and Noesselt 2008; Cecchetti et al. 2016; Dieterich, Endrass and Kathmann 2016; Domínguez-Borràs et al. 2017). This study will therefore focus on how exactly anxiety alters sensory information processing in three distinct sensory modalities while anticipating predictable and unpredictable threats.

By broadening our knowledge on how anticipating different types of threats with our senses occurs in a healthy population, we can better understand the deficits when anxiety becomes a disorder. For this reason, the present study was done on a healthy population in hope that the results will contribute to the development of better treatment for the underlying psychopathology in anxious patients.

To better understand the connection between anxiety, hypervigilance and sensory processing of threats, first one has to understand anxiety. Barlow (2000) described anxiety as a coherent cognitive-affective structure with its core in a sense of uncontrollability focused on possible future threats, danger, or other upcoming potentially negative events. We chose to focus on the component of possible future threats in hope to better understand anxiety as a whole. Unpredictable threat leads to generalized apprehension and hypervigilance (indicating readiness and preparation to deal with a potentially aversive event), whereas predictable threat elicits a "fight or flight" response that is produced by an identifiable threat. Grupe and Nitschke (2013) defined hypervigilance as a state of increased attention to a perceived threat in one's environment. Anxiety is tightly tied to hypervigilance, as it plays a critical role in maintaining it. It is supposed that threat-related arousal facilitates greater attention to a threat and decreases cognitions that would inhibit anxiety (Kimble et al. 2013).

Attention is the process by which the information that we find personally salient reaches our awareness and directs action (Todd and Manaligod 2017). It is the ability to restrict neural processing only to a relevant subset of stimuli while simultaneously excluding task-eligible stimuli from consideration. This usually takes place in the cerebral neocortex (Krauzlis et al. 2018). Anxiety shifts the attention away from a task-directed mechanism to a sensoryvigilance network, that is governed by the amygdala rather than the 
prefrontal cortex (Bishop 2007; Bzdok et al. 2012). The amygdala is a part of the limbic system, a broad area of the forebrain located between the neocortex and hypothalamus that coordinates behavioural and physiological responses to threat-its subcomponents are responsible for processing and integrating environmental information, social information processing, and mounting appropriate attentional, vegetative, and motor responses (Bzdok et al. 2012). It is a highly conserved brain structure that is fundamental to detecting potential danger (Janak and Tye 2015). Hyperactivation of the amygdala can lead to symptoms of depression and anxiety (Yang et al. 2008; Swartz et al. 2015). On the other hand, the prefrontal cortex receives information about all sensory modalities and about the motivational and emotional state of the individual, and can be termed as the brain's "executive" (Niedermeyer 1998; Miller and Wallis 2013). It provides associations between cues and potential threats (Milad and Quirk 2012). Damage done to the prefrontal cortex can cause difficulties in sustaining attention, keeping "on task", and leads to irrational and impulsive behaviour (Miller and Wallis 2013).

\subsection{Anxiety and sensory processing}

The signals of different sensory modalities are processed in anatomically distinct brain areas, and the underlying mechanisms allow them to interact in order to build reliable representations of our environment and to guide our behaviour accordingly (Macaluso 2006). Several sensory-specific areas were identified, responding to stimuli in one or another sensory modality. However, it has been established that signals in different modalities can interact with one another, giving rise to multisensory integration, the study of how information from different sensory modalities can be integrated by the nervous system. Multisensory interactions can affect how we direct attention in space, such as improving our judgment of a visual stimulus by pairing it with a tactile or auditory stimulus (Macaluso 2006). It has been shown to enhance and speed up detection, localization, and reaction to biologically and emotionally significant events (Stein, Stanford and Rowland 2009). Neuroscience has identified multiple "multisensory" brain regions as convergence zones. In those regions neurons receive afferent inputs from several senses and combine them. This multisensory interplay that involves interactions between various sensory modalities can be termed as crossmodality, whereas sensory-specific processes, implying a single unified percept, can be termed as intramodality (Driver and Noesselt 2008).

The brain areas can also respond to specific information independently of the modality conveying the sensory input. This is a supramodal thesis, 
however some authors refer to it as a "task-specific sensory independent" activity (Heimler, Striem-Amit and Amedi 2015), since these supramodal responses do not depend on a specific sensory modality (Cecchetti et al. 2016). An example of a supramodal network are the areas of the brain that process language and integrate visual, auditory, and tactile areas (Lindenberg and Scheef 2007).

It is important to note that authors have been investigating the question of whether stimulus representations from different modalities compete with one another for neural resources. Such competition among modalities would mean that there is evidence of shared crossmodal resources, whereas the lack of competition would point to independent, modality-specific attentional processing (Rapp and Hendel 2003). Research to date indicates that the attentional system can, in fact, be internally organized both into distinct modality specific and crossmodal mechanisms of attention (Rapp and Hendel 2003). Domínguez-Borràs and colleagues (2017) wished to establish whether it is the emotionally significant stimuli that enhance attention and perception when stimuli are simultaneously presented across different sensory modalities. The results showed that emotional modulation heightened sensory reactivity for somatosensory and auditory modality, with no apparent cost to sensory competition. However, visual responses showed a decrease, indicating a mechanism of sensory competition within the visual modality caused by the emotionally significant visual stimulus. This study confirmed and expanded the crossmodal model of attention, which considers that attentional mechanism modulates early modality-specific neural responses across the visual, auditory, and somatosensory cortices, indicating that these effects are controlled by supramodal networks for emotional regulation of perception and attention (networks for sensory response potentiation and sensory response inhibition).

\subsection{Threats and behavioural response}

When the individual is presented with a completely new stimulus, a dilemma is presented-should the stimulus be avoided or approached? If such stimuli are perceptually similar to those associated with danger in the past, they can be evaluated by the individual as potential threats, and the organism responds with defensive responses, such as avoidance or immobility (Grosso et al. 2018). The stress system, the response to and trigger of stressful stimuli, integrates a wide diversity of brain structures which enable us to detect events and interpret them as either real or potential threats, termed stressors (Dedovic et al. 2009). This perception of real or potential threats leads to interaction between mediating molecules with their corresponding receptors 
in the periphery as well as in the brain and results in the stress response, which restores the body homeostasis and promotes adaptation (de Kloet, Joëls and Holsboer 2005; Joëls and Baram 2009). Identification of a stressor, either physical or psychological, leads to the activation of two major constituents of the stress system, the first being the sympathetic-adrenomedullar (SAM) axis, which secretes noradrenaline and norepinephrine, and the second the hypothalamus-pituitary-adrenal (HPA) axis, which secretes glucocorticoids. Once these axes are activated in response to a given threat, they will coordinate a response enabling both an appropriated strategy to deal with threats almost immediately and a homeostasis restoration (Godoy et al. 2018).

The first phase of the response to a potential threat (sympathetic adrenomedullar system-SAM) provides a rapid physiological adaptation resulting in short-lasting responses, such as alertness, vigilance, and appraisal of the situation, and enables the individual to deal with the threat in the initial phase (de Kloet et al. 2005; Joëls and Baram 2009).

When the stress system is not able to overcome the environmental, physiological, or emotional demand, it can become disrupted. This occurs when the demand is extremely strong or chronic and/or during development (Godoy et al. 2018). Chronic stressors in early life can result in permanent epigenetic, endocrine, neural, immune, and inflammatory changes, constituting a relevant risk factor for several neuropsychiatric diseases in adult life (Xiong and Zhang 2013; Berens, Jensen and Nelson 2017). Anxiety disorders tend to be more common in people who experience stress early in life (Fernandes and Osório 2015).

\subsection{Predictable and unpredictable threats in anxiety}

Prediction plays a crucial role in the quality of our life. It allows us to have representations of a particular future event upon which we can produce future-oriented actions, decisions, or behaviours, a network that can be termed anticipation (Pezzulo 2008). In this section I will present current research on the underlying anticipatory attentional mechanism and how neural mechanisms differ with regard to whether we can accurately predict threat in our environment or not.

According to Bidet-Caulet et al. (2012) extraction of informative cues from the surrounding environment occurs through a preparatory mechanism via the deployment of several anticipatory mechanisms-anticipation of imminent threats facilitates the processing of the incoming stimulus, improving the selection and execution of the behavioural response. However, depending on what is expected, different preparation mechanisms are 
involved. Enhanced excitability in visual cortices was observed as a result of the attentional preparation mechanism before the stimulus onset, which induced quicker target detection and processing. Yet when participants expected a target requiring a motor response, the motor preparation mechanism was activated by enhancing excitability in the motor cortices before movement onset.

Neural mechanisms are quite different when we don't know what to expect. Dieterich et al. (2016) examined how attention is allocated under uncertainty about a potential threat. They found expectancy and covariation biases-participants overestimated the frequency of aversive stimuli under uncertainty, while showing a tendency for more negative subjective valences in response to aversive stimuli following uncertain cues. One of the key findings is that uncertainty-induced increase in early attention cannot be modulated by selective processing of neutral and aversive stimuli. This was not specific to visual stimulus processing, which would suggest a generally enhanced attentional response to uncertainly cued targets. These underlying attentional mechanisms produce an ongoing screening for threat in the environment and a continuous elaboration of neutral stimuli which deviate from expectation, indicating the presence of sustained attention for uncertainly cued targets.

\subsection{Event-related potentials}

Event-related Potentials (ERPs) are formed by averaging the EEG measurements of brain responses to repeated presentation of stimuli. They are especially useful for examining rapid processing of potentially threatening stimuli (LeDoux 2000). To further investigate the impact of threat on attention, the current study measured ERP responses elicited by nonpainful electrical stimulation, pictures, and sounds during the NPU-threat task.

We can use the ERP waveform to visualize cognitive operations as they unfold during a trial. We can do this by observing the peaks and troughs of a stimulus-locked ERP. This leads us to ERP components, which can be defined by their polarity (positive or negative voltage), timing, scalp distribution, and sensitivity to task manipulations. A specific component can refer to the underlying cognitive processes and brain activity indexed by the potential (Woodman 2010).

The $\mathrm{N} 1$ is a negative deflection in the ERP waveform that peaks approximately 80-100 ms after the onset of the stimulus at frontocentral electrodes and indicates early perceptual processing of stimuli. It is enhanced when looking at unpleasant in comparison to pleasant and neutral pictures 
(Cuthbert et al. 2000). The enhancement as a result of incoming information is an indicator of increased early vigilance and augmented sensory intake for somatosensory threats (Shackman et al. 2011). We therefore chose to focus on the $\mathrm{N} 1$ as an early indicator of attentional processing in the anticipation and predictability of threat in different sensory modalities and with it explore common neural mechanisms across senses.

Nelson et al. (2015) examined early activation of our defence system in anticipation of no, predictable, and unpredictable shock by observing the P3 and N1 components. Their findings show that the N1 was enhanced at frontocentral sites in anticipation of unpredictable shock and indicate that the increased $\mathrm{N} 1$ may serve as an important component in attention that primes early processing of sensory input when we anticipate possible (unpredictable) danger in our environment. P3 was attenuated, indicating that anticipation of threat in general engages later attention processing. It was one of the first studies done to use startle probe ERPs to demonstrate how the context of a potential threat elicits attentional engagement. They used the NPU-threat task, but included only one modality-the somatosensory.

In a later study Nelson and Hajcak (2017) examined attention in anticipation of threats in two different types of sensory processing-visual and somatosensory. They found that the startle reflex and the participant's ratings of their own feelings on a scale from 1 (not at all anxious) to 7 (extremely anxious) after each condition were greater in anticipation of both types of threat in comparison to no threat, which was further augmented for unpredictable compared to predictable threat. The $\mathrm{N} 1$ was, again, enhanced in anticipation of unpredictable threat in comparison to predictable and no threat in both shock and unpleasant picture trials, meaning that unpredictability enhanced attentional engagement in anticipation of threat in both modalities. However, the shocks elicited greater defensive motivation than unpleasant pictures, which indicates that more intense aversive stimuli are associated with increased effects of unpredictability on the startle reflex. Concerning the neural indicator of the attentional allocation (N1), one of the key findings was that when we compare shocks and unpleasant pictures, the type of threat does not play such an important role as does the potential for threat in general. It is important to note that this study was one of the first to use unpleasant pictures in the NPU-threat task.

Threat-elicited stress is also an important factor that governs our organism's response in a situation when threat is encountered. In an older study Shackman and colleagues (2011) observed early N1 component amplification in participants that were at random exposed to shocks while performing a simple task. This amplification indicates that threat related 
stress (shocks at random) altered attention by amplifying early sensory processing of non-threatening stimuli due to vigilance for threat. At the same time, the later activity of P3 component was attenuated, indicating disrupted task-directed processing. This supports the notion that in anxious anticipation of an uncertain threat, our organism shifts the attention from the prefrontal cortex to the amygdala (Posner 1990; Bishop 2007; Bzdok et al. 2012).

In general, larger $\mathrm{N} 1$ amplitudes that occur in response to nonthreatening stimuli have been found in populations prone to high levels of anxiety, such as panic attacks (Wise et al. 2009), social anxiety (Felmingham et al. 2016), and PTSD (Zukerman et al. 2018). The N1 was specifically enhanced in those with PTSD in a study done by Zukerman et al. (2018) when participants were presented with the auditory novelty oddball paradigm to discriminate between target, novel, and standard tone. Such findings are especially indicative of hypervigilance of early automatic attention.

\subsection{The aim of our study}

The goal of our study was to investigate sensory processing during anticipation of predictable and unpredictable threats in three different modalities (auditory, visual, and tactile). Specifically, the emphasis was on the neutral stimuli presented after the cue and just before the threat to capture the neural mechanisms of anticipation. We wished to examine if the state of hypervigilance alters the attention to neutral stimuli in any of the three modalities. In addition, we wanted to establish if sensory processing is selectively influenced during anticipation of threat. Our main research question was how anticipation of threat affects sensory responses in the auditory, visual, and somatosensory modalities.

There has been a proliferation of studies on the connection between emotionally significant stimuli and sensory processing and attention. With predictable threats, the brain mechanisms work through several anticipatory mechanisms depending on what is expected (Bidet-Caulet et al. 2012), which is different from uncertainty-induced early attention, since the latter is not selectively influenced but generally enhanced to uncertainly cued targets. Stimulus that is presented after an uncertain cue (either neutral or aversive) tends to elicit an enhancement of early phasic and sustained attention, a neural indicator observable with the N1 component (Dieterich et al. 2016). These targets are processed through different sensory processing channels. Here, the results of previous studies have yielded inconsistent findings. Some argue that the sensory modality does not play a crucial differentiating role in the $\mathrm{N} 1$ enhancement, and that different types of aversive stimuli produce 
comparable attentional engagement (Nelson and Hajcak 2017). Others say that due to sensory competition, the type of threat is an important factor in attentional allocation in the anticipation of unpredictable threat. Sensory effects that would be irrelevant in neutral conditions may become more salient in a threatening environment, only when these events do not compete within a sensory channel (Domínguez-Borràs et al. 2017). However, the amplification of the $\mathrm{N} 1$ component is an indicator of early attentional engagement (vigilance for threat) caused by the anticipation of unpredictable threat (Shackman et al. 2011; Nelson et al. 2015; Nelson and Hajcak 2017).

A review of literature on this subject has led us to formulate the following hypotheses:

H1: When anticipating unpredictable threat, hypervigilance in all three sensory modalities can be observed as the enhancement of the N1 component.

H2: The enhancement of the N1 component in all three sensory modalities in anticipation of unpredictable aversive stimuli compared to predictable aversive stimuli or no aversive stimuli proves the presence of supramodality, suggesting a sensory-independent activity.

H3: The enhancement of the N1 component for neutral stimuli of the same sensory modality as the aversive stimuli proves the presence of intramodality, suggesting a sensory-dependent activity.

\section{Methodology}

\subsection{Participants}

Subjects (7 male, 22 female) were undergraduate Psychology students at the Erasmus University of Rotterdam who obtained course credits in exchange for the participation. The subjects applied to participate in the study through a website of the Erasmus University of Rotterdam designed specifically for students to apply for all the ongoing research studies on the university. All participants provided written informed consent and the research protocol was approved by the Ethical Comittee of Erasmus University of Rotterdam. The social demographics of the sample are presented in Table 1. The data of one female participant, who was feeling anxious during the experiment and decided to withdraw, was excluded. The final number of participants was 28. Exclusion criteria were psychiatric or neurological disorders (for example epilepsy). The participants were informed about the conditions that would 
distort the EEG data (hair gel, any kind of skin make-up ...) and asked to avoid them on the day of the experiment.

The sample is rather small and not completely representative. It is not balanced in gender and it comprises only students. Due to these factors the results of the study cannot be generalized to the whole of population (see Discussion).

Table 1: Social demographics

\begin{tabular}{|l|l|c|c|c|c|c|}
\hline & & N & M & SD & Min & Max \\
\hline Age & Female & 22 & 20.82 & 2.91 & 18 & 29 \\
& Male & 6 & 20.83 & 1.60 & 18 & 23 \\
\hline Nationality & Dutch nationality and origin & 7 & & & & \\
& Dutch nationality and other & 2 & & & & \\
& descent & & & & & \\
& Other nationality and origin & 19 & & & & \\
& & & & & & \\
\hline
\end{tabular}

Source: Author

\subsection{Apparatus and measures}

\subsubsection{Stimuli}

For the auditory neutral stimulus we used a beep tone with a fundamental frequency of $500 \mathrm{~Hz}$, played at a volume of $70 \mathrm{~dB}$. For auditory aversive stimulus we used the sound of a woman screaming at $90 \mathrm{~dB}$. For somatosensory neutral and aversive stimulus we used electrical stimulation at individual pain threshold administered to the left lower arm muscle (M. Brachioradialis); the neutral and aversive stimulus differed in their duration as described below (see Procedure). Gabor patches were used for visual neutral stimulus as seen in Figure 1. For visual aversive stimulus we selected a picture from the International Affective Pictures System that presented a murdered man as seen in Figure 2 (IAPS; Lang et al. 2008). The visual stimuli and cues had a size of $500 \times 500$ pixels.

The stimuli were presented to the participants in an isolated room with a screen at a viewing distance of approximately $1 \mathrm{~m}$. The screen size was 1024 x 768 pixels on a $22^{\prime \prime}$ screen. 
Figure 1: Neutral visual stimulus

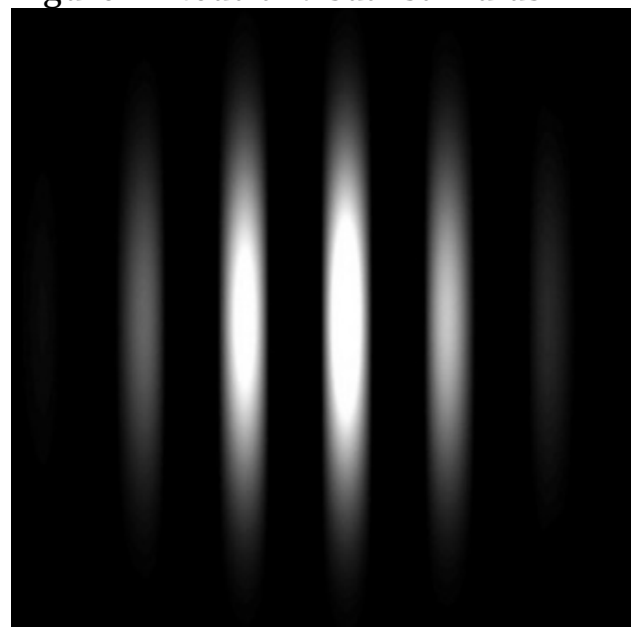

Source: IAPS; Lang et al. 2008

Figure 2: Aversive visual stimulus

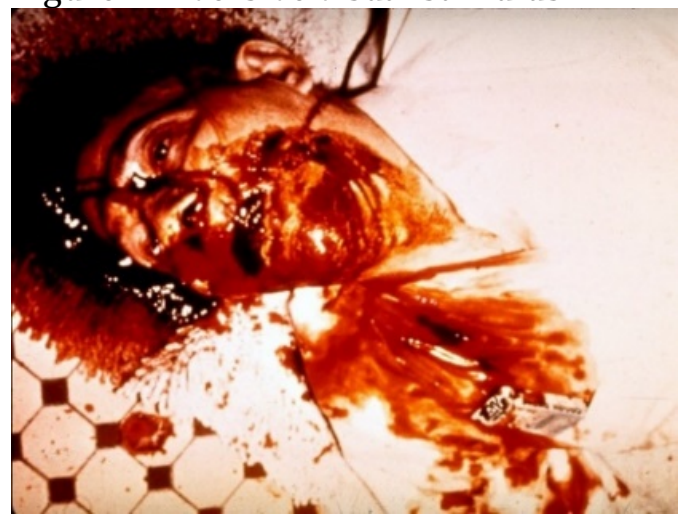

Source: IAPS; Lang et al. 2008

\subsubsection{Questionnaires and Tests}

Before the experiment, the participants filled out the State-Trait Anxiety Inventory (Spielberger et al. 1983) (STAI) questionnaire and the Beck Depression Inventory-BDI (Beck 1961) on a computer. STAI was used to measure state anxiety (current state of anxiety) and trait anxiety (anxiety on a personal level). 20 items are allocated to each the state or trait anxiety, respectively. Responses for state anxiety assess feelings "at the moment. The responses for trait anxiety scales asses frequency of feelings. Higher scores 
are positively correlated with higher levels of anxiety. The BDI measure is a 21-item scale with a good internal reliability and consistency. It also picks up anxiety states to some extent and focuses less on the somatic aspects of depression (Beck 1961).

The anxiety scores from the STAI questionnaire (Spielberger et al. 1983) were within the normal range in all cases (STAI-State: $\mathrm{M}=38.6$ vs 38.8 in standard population, $\mathrm{SD}=10$; STAI-Trait: $\mathrm{M}=42.4$ vs 40.4 in standard population, $\mathrm{SD}=8.2$ ).

We included one more written test after the experiment in which the subjects estimated the probability of a threat occurring after each cue. The purpose was to verify whether the subjects understood and followed the cues throughout the experiment.

\subsubsection{EEG equipment}

Continuous EEG was recorded using an elastic cap with 64 sintered $\mathrm{Ag} / \mathrm{AgCl}$ electrodes placed according to the international 10/20 system (Oostenveld and Praamstra 2001). An electrooculogram was recorded using four additional facial electrodes: two placed approximately $1 \mathrm{~cm}$ outside of the right and left eyes and two placed approximately $1 \mathrm{~cm}$ above and below the left eye. Data was recorded using the BioSemi ActiveTwo amplifier.

Signals were recorded with a low-pass filter of $134 \mathrm{~Hz}$ and were digitized with a sample rate of $512 \mathrm{~Hz}$ and 24-bit analog/digital conversion. BioSemi uses the common mode sense (CMS) and driven right-leg electrodes to create a feedback loop that replaces the conventional ground electrode. The CMS was used as an online reference.

\subsection{Procedure}

Participants first read and signed the consent form and then completed the STAI and BDI questionnaires on the computer which took approximately 10 minutes. The next phase of the experiment took place in an isolated room where the EEG recording took place. When the EEG was set up, we proceeded with the pain thresholding procedure which lasted for approximately 10 minutes. Each individual's pain-threshold was measured to define the intensity of the somatosensory stimuli. We used an electric stimulus with a frequency of $50 \mathrm{~Hz}$ generated by a constant current stimulator (Digitimer DS7A, Digitimer LTD., Welwyn Garden City, UK). Prior to the pain thresholding procedure a scale from 0 to 10 was presented to a subject, 4 indicating "just noticeable pain". Participants received increasing levels of shock, with 2 series of increases of the electrical stimulation and 2 series of decreases, starting with $0 \mathrm{~mA}$. The direction changed each time a subject 
rated the shock as 4 . The electrical current increased and decreased always by $1 \mathrm{~mA}$. To determine the pain threshold we took the electric charges rated as 4 , calculated the mean and added $30 \%$. The electrical stimulation was always applied once for the duration of $2 \mathrm{~ms}$. The mean shock intensity level across the entire sample was $2.34 \mathrm{~mA}(\mathrm{SD}=1.65)$ and the rating $5.72(\mathrm{SD}=$ 0.97).

After the pain thresholding procedure, the subjects were explicitly instructed about the predictive value of cues. Next, they had to rate neutral and aversive stimuli, measuring their intensity and unpleasantness. The question "How intense do you find this stimulus?" was displayed on the computer screen, after which the stimuli were presented one by one. The participants used a cursor to choose the perceived value on a digital scale. This procedure was repeated for the question "How unpleasant do you find this stimulus?". The overall range of the scale was from -50 on the left (the least intense or unpleasant) and 50 on the right (the most intense or unpleasant). They rated the stimuli before (Pre) and after (Post) the experimental session.

Figure 3: The procedure of the experiment-the length of each box presents the approximate duration of each step

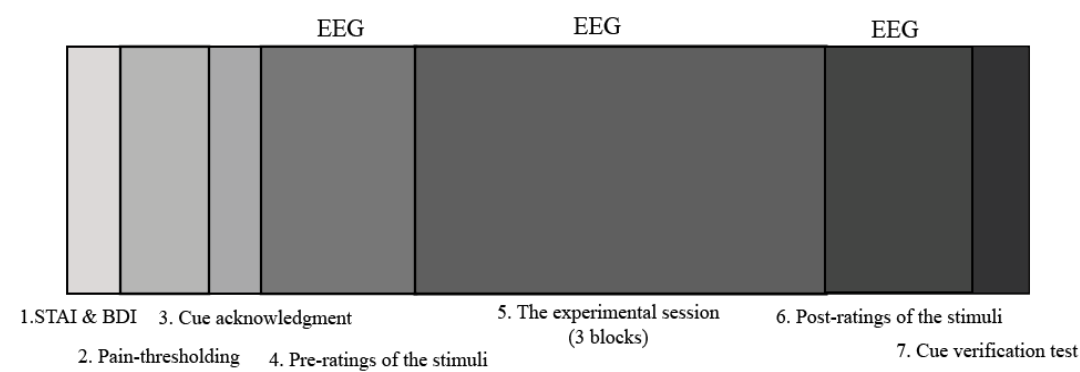

Source: Author

For the three blocks we used the NPU-threat task (Schmitz and Grillon, 2012). We had one condition with three levels: $100 \%$ no threat, $100 \%$ predictable threat, and unpredictable threat (50\% threat, 50\% no threat). Each block had a different threat modality-one with the aversive sound stimulus (woman screaming), the second with the aversive visual stimulus (Figure 2) and the third with the aversive somatosensory stimulus (long electric stimulation). Blocks were presented in one of the following orders (counterbalanced): 123, 231 or 312. 
Figure 4: A schematic representation of an experimental trial

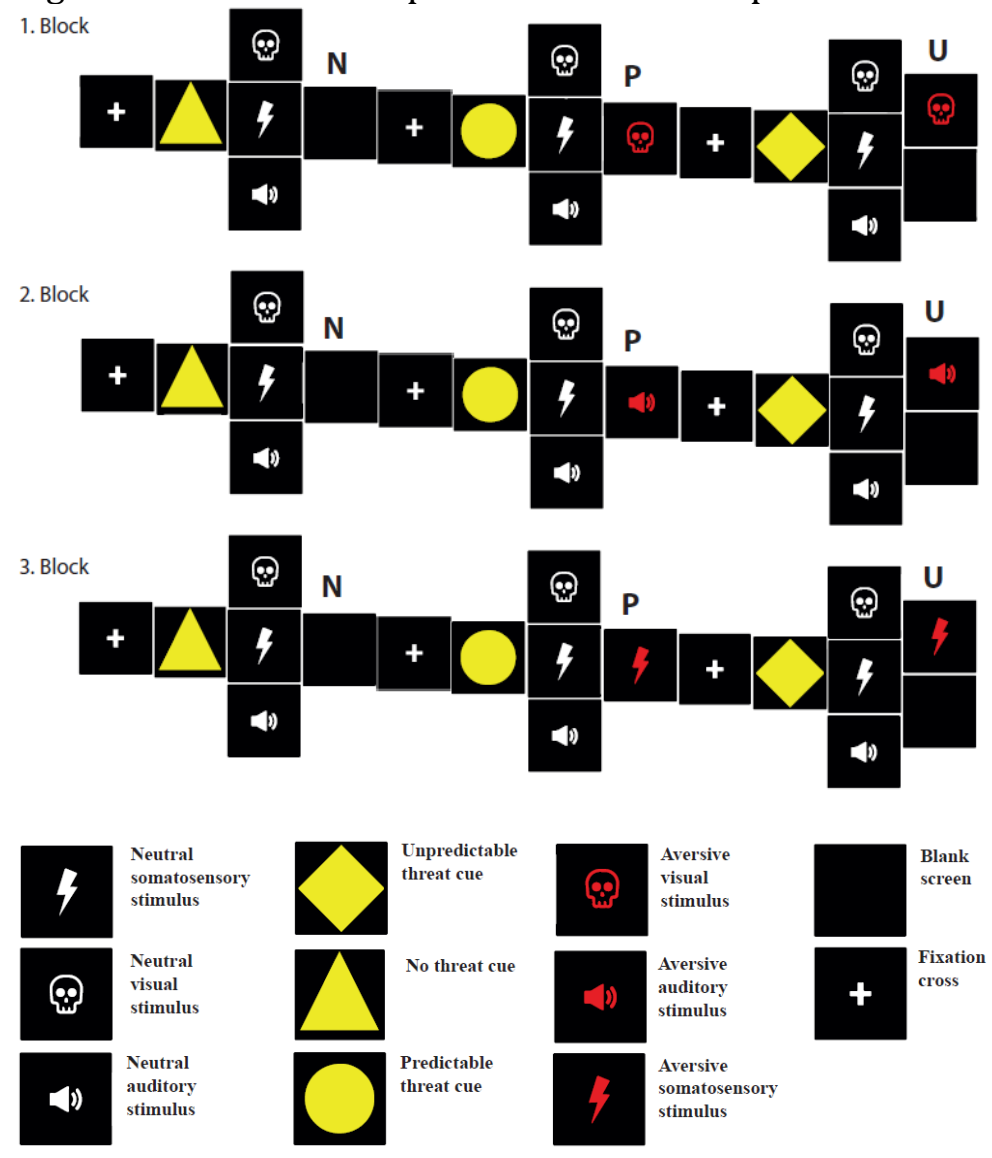

Source: Author

Figure 4 is a schematic representation of an experimental trial. The triangle, circle, and diamond represent the cues, the white images represent neutral stimuli, and the red images the aversive stimuli. In the no threat condition, no aversive stimulus was presented, leaving the participant with a blank screen. In the predictable threat condition, participants received one of the aversive stimuli, depending on the block. In the unpredictable threat condition, the participants got either a blank screen (no threat) or an aversive stimulus (threat).

Each NPU condition contained 20 trials, which amounted to 540 trials in total, i.e. 180 trials per block and 60 per conditions that were randomized. A trial began with a fixation cross followed by a cue in the form of a geometric 
shape indicating the condition. Then the neutral stimulus was presented three times, followed by either no threat or predictable/unpredictable threat. The three blocks lasted for the duration of approximately 50 minutes.

Figure 5: An example of the no threat trial and the predictable threat trial

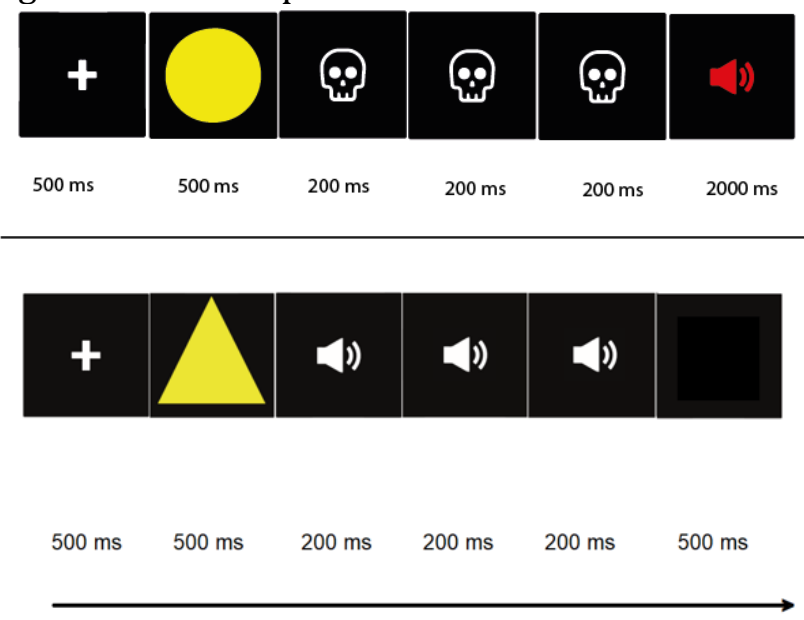

Source: Author

Cues indicating the NPU condition lasted for $500 \mathrm{~ms}$. The neutral visual stimulus and the neutral auditory stimulus lasted for $200 \mathrm{~ms}$ and were repeated for 3 times, with variable inter-stimulus intervals (1000-2000 ms). The neutral somatosensory stimulus lasted for $2 \mathrm{~ms}$. The aversive visual and auditory stimuli lasted for $2000 \mathrm{~ms}$ and the aversive somatosensory stimulus lasted for 10 times $2 \mathrm{~ms}$ on and $2 \mathrm{~ms}$ off $(40 \mathrm{~ms}$ ).

After the three blocks, participants rated the neutral and aversive stimuli in the same way as they did at the beginning. The final task of the entire experiment was a short cue verification test where participants were asked to write down the probability of a threat occurring after each cue. The whole experiment lasted for approximately 2 hours.

\subsection{Data Analysis}

We conducted the EEG analysis using the BrainVision Analyzer 2.1 software package (Brain Products GmbH, Munich, Germany).

Data was referenced offline to an averaged reference, band pass filtered ( 0.1 to $30 \mathrm{~Hz}$ ), and corrected for eye movement artifacts using the Gratton method (Gratton, Coles and Donchin, 1983). Data were segmented with 
regard to neutral stimulus onset ( $-200 \mathrm{~ms}$ onset to $400 \mathrm{~ms}$ ). Finally, an artifact correction (exclusion of epochs exceeding $>150 \mathrm{mV}$ amplitude change or low activity) and baseline correction (200 ms) was applied.

We focused on the $\mathrm{N} 1$ component elicited by the neutral stimuli. The time windows and the topographic activity at that time can be seen in Figure 6. For the neutral auditory stimulus we extracted the mean amplitude in the time window of 140-160 ms scored over C1, CZ, and C2 on the basis of visual inspection (grand average across all auditory stimuli). For the neutral visual stimulus we extracted the mean amplitude in the time window of 150-180 ms. For the left hemisphere we pooled the P07, P7, and P9 and for the right hemisphere P08, P8, and P10. For the neutral somatosensory stimulus we extracted the mean amplitude in the time window of 105-135 ms, scored over $\mathrm{Cz}, \mathrm{C} 2$, and $\mathrm{C} 4$. Since the electrode for electrical stimulation was placed on the left arm, the selected electrodes were predominantly on the right hemisphere due to contralateral processing.

The values of mean amplitudes of neutral stimuli were then exported into the IBM SPSS Statistics, version 23 (2012). Separate grand averages were conducted for each type of threat (visual, auditory, somatosensory) and each condition (no threat, predictable threat, unpredictable threat).

The new clusters for N1 amplitudes were then analyzed using the repeated-measures ANOVA. Separate analyses were conducted for each modality. Significant interactions were followed up by post-hoc $t$ tests with the criteria of $\mathrm{P}<0.05$.

Figure 6: Topographic maps of the N1 activity
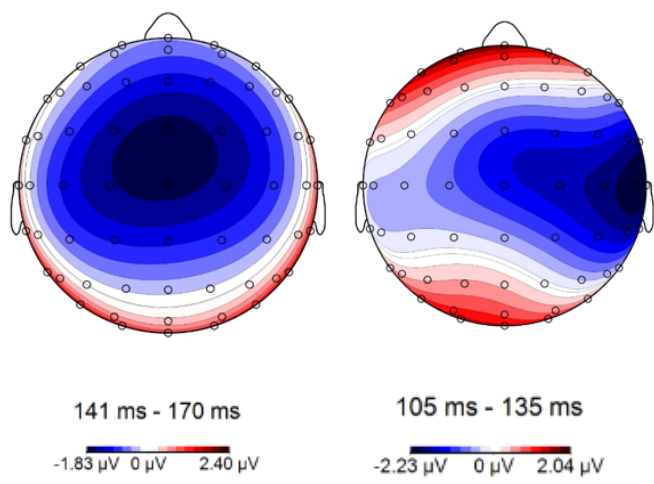

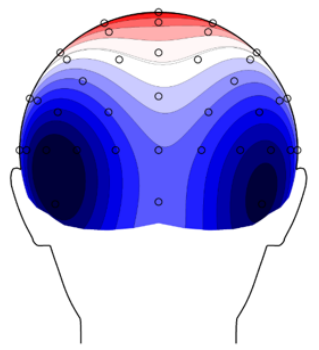

$150 \mathrm{~ms}-180 \mathrm{~ms}$

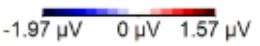

Source: Author 
The ratings of the stimuli were also exported into the IBM SPSS Statistics, version 23 (2012). The location of the cursor on the digital scale presented the value from -50 to 50 . A 2 (aversive stimuli, neutral stimuli) x 3 (visual, auditory, somatosensory) and 2 (pre-experiment, post-experiment) $\mathrm{x} 2$ (aversive stimuli, neutral stimuli) x 3 (visual, auditory, somatosensory) repeated measures ANOVAs were done on the intensity and unpleasantness ratings.

\section{Results}

\subsection{Stimuli ratings}

A 2 (neutral, aversive) x 3 (visual, auditory, somatosensory) repeated measures ANOVA on the intensity $\mathrm{F}(2,54)=11.55, \mathrm{p}<.001$ and unpleasantness ratings $F(2,54)=14.73, p<.001$ yielded a significant main effect of the aversive sound stimulus, indicating it was more unpleasant and intense than the aversive somatosensory and the aversive visual stimulus. This effect is presented in Figures 7 and 8.

Another repeated measures ANOVA 2 (pre-experiment, postexperiment) x 2 (neutral, aversive) x 3 (visual, auditory, somatosensory) on stimuli ratings yielded significant results - the aversive visual stimulus was less unpleasant after the experiment and that the aversive auditory stimulus sounded more unpleasant after the experiment $F(2,54)=4.45, p=.016$. The timeline of the ratings through the experiment can be seen in Figure 9.

Figure 7: Intensity ratings for neutral and aversive stimuli in all three modalities

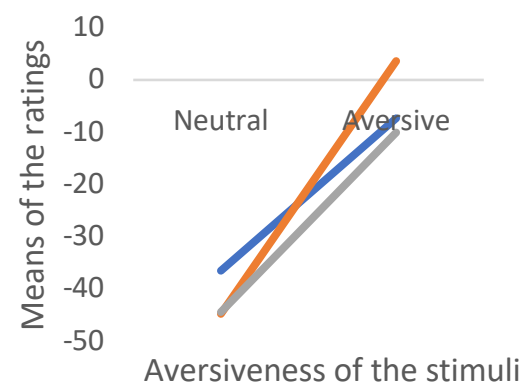

-Shock $\longrightarrow$ Sound $\longrightarrow$ Picture

Source: Author 
Figure 8: Unpleasantness ratings for neutral and aversive stimuli in all three modalities

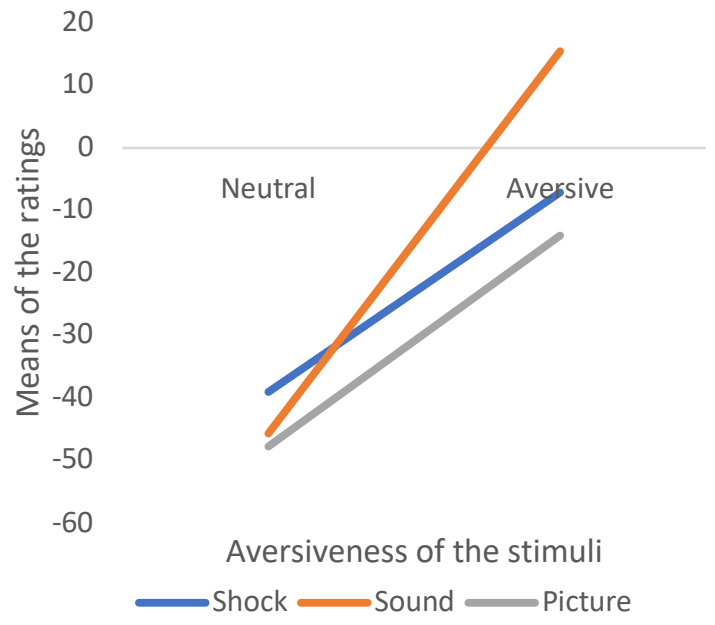

Source: Author

Figure 9: Unpleasantness ratings before and after the three blocks

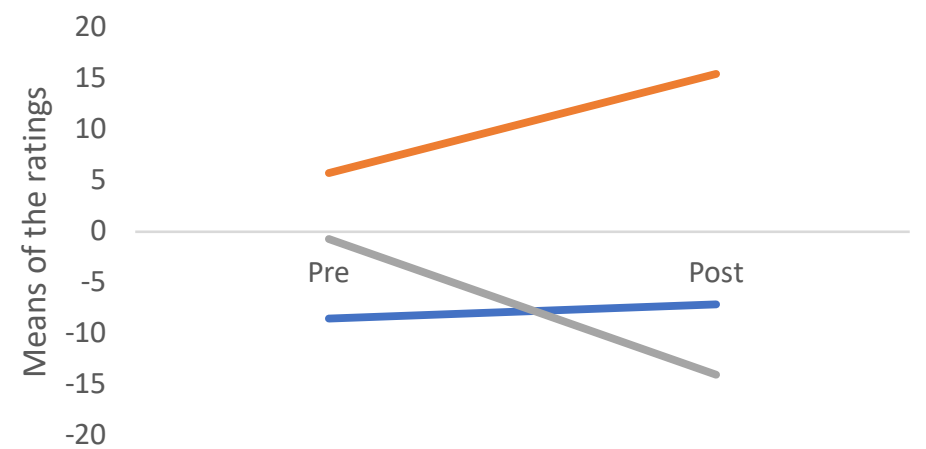

Before and after the experiemental session

Shock Sound $\longrightarrow$ Picture

Source: Author 


\subsection{N1 amplitudes}

A repeated measures ANOVA with three within-subject factors was performed to analyze the visual modality. A 2 (left hemisphere, right hemisphere) x 3 (unpredictable, predictable, no threat) x 3 (auditory, visual, or somatosensory threat) repeated measures ANOVA on the N1 amplitude yielded no significant main effect or interaction $F(4,108)=1.52, p=.201$.

Another repeated measures ANOVA was performed with 2 within-subject factors in order to analyze the auditory modality. The 3 (unpredictable, predictable, no threat) x 3 (auditory, visual, or somatosensory threat) for the auditory modality repeated measures ANOVA did not yield any significant main effect or interaction $\mathrm{F}(4,108)=2.19, \mathrm{p}=.074$. These results indicate that the $\mathrm{N} 1$ amplitude in the visual and auditory modality did not significantly differ between threat and neutral modality nor was there any interaction.

The 3 (unpredictable, predictable, no threat) x 3 (auditory, visual, or somatosensory threat) repeated measures ANOVA on the somatosensory modality yielded no significant main effect indicating that the $\mathrm{N} 1$ amplitude does not differ between the aversive and neutral modality. Nevertheless, there was a significant interaction between the aversive visual stimulus and the aversive somatosensory stimulus in the unpredictable condition $F(4,108)$ $=2.92, \mathrm{p}=.024$ as seen in Figure 10. Because of this we performed a t-test to investigate the correlation. The correlation between the no threat condition and the unpredictable threat condition yielded significant results $\mathrm{t}(27)=2.39$, $\mathrm{p} .024$, as did the correlation between the no threat condition and the predictable threat $\mathrm{t}(27)=2.47, \mathrm{p} .02$.

Figure 10: Scores of the N1 component in neutral somatosensory stimulus presented with threat in all three modalities and with all three conditions

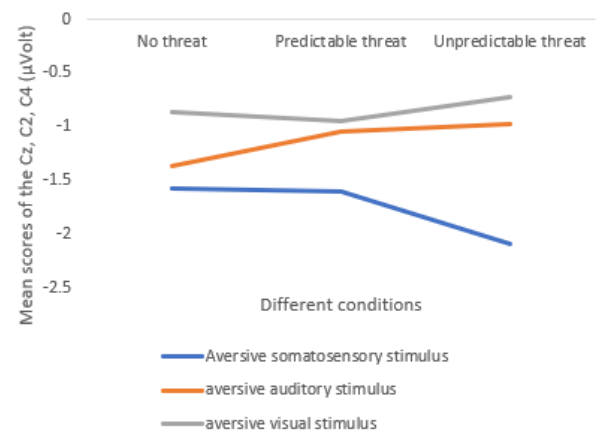

Source: Author 
In Table 2 we can see the scores for the average of the three electrodes $(\mathrm{Cz}$, $\mathrm{C} 2$, and $\mathrm{C} 4$ ) for the neutral somatosensory stimulus in different conditions (the no threat, predictable threat and unpredictable threat) and different threat modalities (auditory, visual and somatosensory). We can see that the unpredictable threat with somatosensory modality is the lowest. This is also well presented in Figure 13.

Table 2: descriptive statistics for the N1 component

\begin{tabular}{|l|c|c|}
\hline & Mean & Std. Deviation \\
\hline No threat, somatosensory modality & -1.58 & 2.16 \\
\hline No threat, auditory modality & -1.37 & 1.80 \\
\hline No threat, visual modality & -.87 & 2.21 \\
\hline Predictable threat, somatosensory modality & -1.61 & 1.84 \\
\hline Predictable threat, auditory modality & -1.05 & 1.58 \\
\hline Predictable threat, visual modality & -.95 & 1.53 \\
\hline Unpredictable threat, somatosensory modality & -2.09 & 1.95 \\
\hline Unpredictable threat, auditory modality & -.98 & 1.73 \\
\hline Unpredictable threat, visual modality & -.72 & 1.92 \\
\hline
\end{tabular}

Source: Author

Figures below display N1 waveforms in all three conditions when the neutral somatosensory stimulus was presented in all three different threat modalities. The black waveform is the averaged and postprocessed ERP waveform of the N1 component in the safe condition, when no threat was presented. The blue waveform indicates the $\mathrm{N} 1$ component when the threat of either auditory, visual or somatosensory modality was predictable. The red waveform stands for the $\mathrm{N} 1$ component when the threat in all three modalities was unpredictable. The enhancement of the $\mathrm{N} 1$ component in the unpredictable condition compared to predictable and neutral conditions with the somatosensory threat is clearly visible. 
Figure 11: The N1 component in the auditory threat

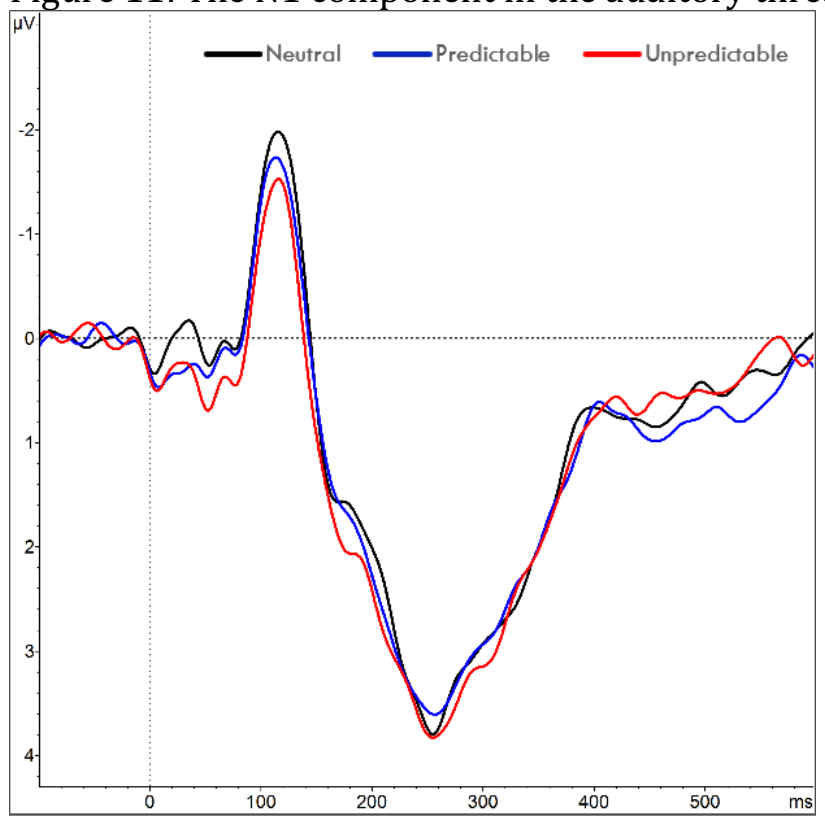

Source: Author

Figure 12: The N1 component in the visual threat

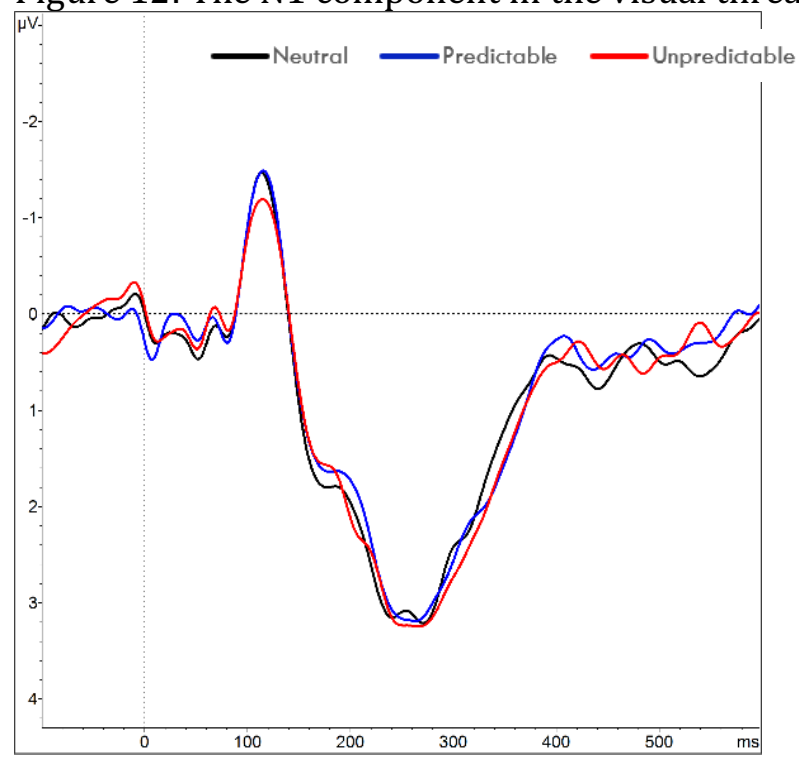

Source: Author 
Figure 13: The N1 component in the somatosensory threat

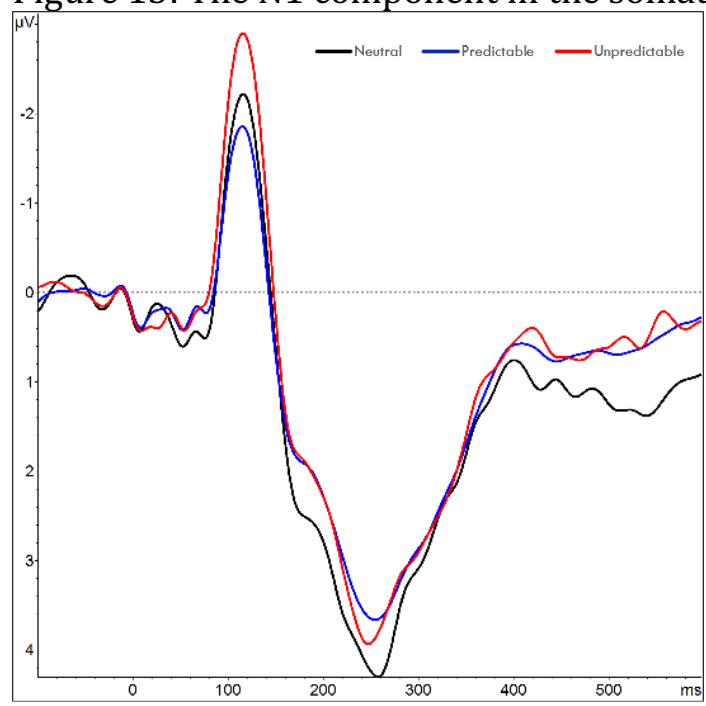

Source: Author

\section{Discussion}

The aim of this study was to investigate the influence of threat anticipation on sensory responses in the visual, auditory, and somatosensory modalities. We analyzed the $\mathrm{N} 1$ component when neutral stimuli were presented and compared the amplitudes of different modalities in the no threat, predictable threat, and unpredictable threat conditions. We took ratings of the neutral and aversive stimuli before and after the three blocks of different threat modality to distinguish between different ranges of their intensity and unpleasantness and to observe whether habituation was present. We also checked for any possible correlation between the BDI, STAI results and the $\mathrm{N} 1$ enhancement to see if there was any interference with our results.

As we did not find the $\mathrm{N} 1$ enhancement in any of the three modalities in the unpredictable condition, we can dismiss our first hypothesis along with the second supramodal hypothesis. We did not find a significant effect on the $\mathrm{N} 1$ amplification in the visual modality while anticipating the unpredictable threat, indicating that hypervigilance did not occur. A possible explanation would be that in contrast to shock, which presents a source of actual physical threat, the aversive picture represents a mere visual threat (Lissek et al. 2007). It is noteworthy that some potential effects of habituation were present in the ratings of the stimuli, specifically that the aversive visual 
stimulus appeared less unpleasant after the three blocks. Such habituation is commonly observed if emotional stimuli are repeated over successive trials (Plichta et al. 2014). There was also no significant effect on the N1 amplification in the auditory modality while anticipating the unpredictable threat, however the interaction between threat condition and the auditory modality was barely insignificant. This could be explained by a fairly small sample of 28 participants. A larger sample would provide more trials, which could lead to better signal-to-noise ratios (Dieterich et al. 2016). The stimuli ratings also show that the aversive auditory stimulus appeared more unpleasant and intense than the aversive somatosensory and visual stimuli, supporting the notion that significant results would be possible with a larger sample.

The results showed that only anticipating an unpredictable aversive somatosensory stimulus had a significant effect on sensory responses to neutral somatosensory stimuli. This is evident from the significant interaction between threat modality and condition. Shocks appear to be a more potent elicitor of vigilance mode (Shackman et al. 2011), a state in which N1 amplification can be observed due to the modulation of extrastriate cortex by the amygdala (Lim, Padmala and Pessoa 2009; Bzdok et al. 2013). This supports the notion of a heightened vigilance to somatosensory stimuli while anticipating a somatosensory threat. Because there was a significant effect on sensory responses to neutral stimuli in the condition where threat was of the same modality, we can partially confirm the third, intramodal hypothesis, concerning the notion of a sensory-specific brain organization according to function (Driver and Noesselt 2007). However, this should be considered with caution since source localization remains imperfect with EEG (Woodman 2010) and we are witnessing a growing body of literature on the emerging consensus that multisensory effects can influence traditional "sensoryspecific" regions (Driver and Spence 2000; McDonald, Teder-Salejarvi, and Hillyard 2000; Vroomen and Gelder 2000; Frassinetti, Bolognini and Làdavas 2002; Lovelace, Stein and Wallace 2003; Macaluso 2006). Our results are somewhat inconsistent with previous findings. Domínguez-Borràs and colleagues (2017) posit that emotional processing is similar to attentional processes that modulate early modality-specific neural responses across the visual, auditory, and somatosensory cortices, thereby extending the crossmodal hypothesis. They observed functional connectivity as an increase in activity in areas responsible for auditory and somatosensory information processing that was concurrent with a decrease in areas responsible for visual information processing when participants were presented with fearful faces. The difference in our results could be attributed to spatial proximity between 
stimuli in the study by Domínguez-Borràs (all the stimuli were near the subjects' head), which is known to influence crossmodal attention (Driver and Spence 2000). That was not the case in our study since none of our stimuli were close to the subjects' head. There is an increasing body of literature supporting brain connectivity, especially the functional connectivity, which postulates temporal dependency of neuronal activation patterns of anatomically separated brain regions (Lang et al. 2012). The fact that we did not observe crossmodal, functional connectivity does not exclude the latter, but rather highlights the limitations of our study, described below.

Our results showed no sensory competition between auditory, visual, and somatosensory modalities, which is why we were able to observe independent, modality-specific attentional processing, but not functional connectivity (Rapp and Hendel 2003). With this in mind we can, again, partially confirm our intramodal hypothesis. Domínguez-Borràs and colleagues (2017) observed a mechanism of sensory competition through a decrease in primary visual cortex due to concomitant processing of emotional faces and irrelevant visual stimuli, however these findings are inconsistent with our own. It should be noted that peripheral distractors and emotional faces appeared concurrently, whereas the stimuli presented in our study were nonconcurrent. A possible explanation would be the unequal aversiveness of our stimuli. Neutral conditions may appear more salient in a threatening environment (Domínguez-Borràs et al. 2017) and it could be that some of our aversive stimuli were not threatening enough. We cannot conclude, however, that different types of aversive stimuli produce comparable attentional engagement (Nelson and Hajcak 2017), due to the fact that we observed the $\mathrm{N} 1$ enhancement only in one sensory modality. Note that sensory competition implicates both the low-level sensory systems and the high-level fronto-sensorimotor networks. A study done by Huang et al. (2015), investigating the multisensory competition in the sensory pathway interactions with fronto-sensorimotor and default-mode network (DMN) regions, presented their participants simultaneously with visual and auditory targets. They found increased prestimulus activity in the prefrontal cortex and decreased prestimulus activity in the DMN, which enhanced visual dominance over auditory modality while exploiting the fMRI. The DMN is a concept that describes a resting state activity in which an individual is awake and alert, but not actively involved in an attention demanding or goal-directed task. The network includes precuneus/posterior cingulate cortex (PCC), medial prefrontal cortex (MPFC), and medial, lateral, and inferior parietal cortex (Lang et al. 2012). Huang et al. (2015) also observed that the ERPs and the divergence between the two conditions began to reach significance at 250 
ms after the onset of the target. A possible explanation why we did not observe any sensory competition could therefore be our choice of method as the DMN network was more difficult to observe with the electrodes we chose. We also focused on the $\mathrm{N} 1$ component that peaks at approximately $100 \mathrm{~ms}$.

As mentioned, a probable explanation for our results could also be our choice of electrodes. The fact that the right hemisphere is involved in vigilance and arousing states of anxiety is well documented (Posner 1990; Shackman et al. 2011). For example, in their study, Shackman et al. (2011), used a sophisticated method of determining the most stress-sensitive scalp regions where they chose a large cluster of electrodes centred along fronto-central part of the scalp and in the vicinity of the right mastoid. States of stress elicited by threat of shock and aversive images potentially activate the right lateral prefrontal cortex (Dalton et al. 2005; Dolcos 2006; Coan, Schaefer and Davidson 2006). A study done by Gilmore, Clementz, and Berg (2009) specifically investigated hemispheric laterality and ERP components. The results showed consistently stronger target-specific cortical activity in right temporal and parietal areas when the participants performed the auditory oddball paradigm binaurally and monaurally. The N1 component occurs only at $80-100 \mathrm{~ms}$ post stimulus, which is why it is very important to choose the right electrode (Woodman 2010). It could be that we did not get more significant results because our electrodes were mainly positioned around the central site of the scalp.

There are other factors that could interfere with our results. For example, the results of the BDI and STAI questionnaires showed that all our participants were in the normal range. There was no correlation between the results of the questionnaires and different scores of the $\mathrm{N} 1$ component between different conditions, indicating that the final average scores of the N1 component were not influenced by the symptoms of anxiety or depression. Furthermore, the participants took a test after the three blocks where they had to indicate the probability of a threat occurring after each cue. This test served as a verification whether they understood the meaning of cues throughout the experiment, which was important to differentiate between the three different conditions. The results indicated that the majority accurately followed cues throughout the experiment, while some did not. One participant indicated that the probability of a threat occurring after the predictable cue was $50 \%$, four participants indicated the wrong probability of a threat occurring after the unpredictable cue, and six participants indicated the wrong probability of a threat occurring in the safe condition. These participants misunderstood the cues and it could be they were not 
paying close attention to the experiment, which could have interfered with our results by diminishing the number of validate trials.

The present study has several limitations that should be taken into consideration. First, the generalizability of the results to other populations (such as children or clinical populations) is questionable due to the fact that the sample consisted of college undergraduates. Second, a small sample size, as was the case with our experiment, has low statistical power, whereas a larger sample could produce more significant results overall. Third, our study contained disproportionate aversiveness of different stimuli and threats therefore did not appear threatening on the same level. Another possible limitation could be the environment of the experiment-a laboratory, located at the university where participants study, an environment that is familiar to them and overall safe. Furthermore, the experiment was always led by two of our team, students as well, meaning that the participants had a sense of shared social identity. The results of the state anxiety were also within normal range. These factors could have attenuated the threats that consequently simply did not appear "threatening enough". Future studies should involve equally threatening stimuli and strive to use stimuli on a similar level of arousal, as it is well known that arousal drives attention potentially captured by ERP components (Cuthbert et al. 2000; Schupp et al. 2000; Schupp et al. 2007; Weinberg and Hajcak 2010). Considering the above, a more appropriate design for future studies would be to conduct them individually with separate modalities and use a homogenous category of stimuli within the same modality. This is especially plausible when the NPU-threat task is used to reduce the element of uncertainty during the predictable threat condition (Nelson et al. 2015). Nonetheless, our results add to the growing number of studies that use the NPU-threat task, indicating that measures of attention during the NPU-threat task have valuable psychometric properties.

\section{Conclusions}

Sensory processing in a hypervigilant state, i.e. when an individual experiences increased attention to a possible threat in the environment, is still not completely understood. The present study aimed to examine sensory processing during anticipation of predictable and unpredictable threats in the auditory, visual, and somatosensory modalities. We were interested in neural responses to neutral stimuli preceding threat. Furthermore, we focused on three modalities to see if the processes are selectively influenced in the course of anticipating threat. This study sought to contribute to a better 
understanding of hypervigilance and thereby expand our knowledge of the anxious state.

The results showed the $\mathrm{N} 1$ enhancement in the unpredictable condition in the somatosensory modality. Sensory responses to neutral stimuli were enhanced when presented with the unpredictable threat of shock. These results imply that an individual goes into a hypervigilant state when there is a possibility of a somatosensory threat in the environment. We did not observe any presence of sensory competition, meaning that processes were not selectively influenced. As we detected an enhanced N1 in the neutral stimuli that was of the same modality as the aversive threat, we can confirm the intramodal hypothesis that suggests a sensory-dependent attentional activity.

This is somewhat inconsistent with previous findings. In recent years, crossmodality, along with functional connectivity of the brain, has been studied to a considerable degree. However, it is possible that with a larger sample we would also observe the $\mathrm{N} 1$ enhancement in the auditory modality, as the results were barely insignificant. Overall, future studies should focus on a larger sample and a modality-specific design of the study in combination with a homogenous category of stimuli, which could produce findings that would be more consistent with recent literature.

Nevertheless, the present study contributes to the growing body of literature on how unpredictable threat leads to a more sustained level of anxiety, producing a state of increased attention to stimuli that would otherwise appear non-threatening. A better understanding of the origin of unpredictability and its consequences could provide clues to identifying unpredictability as a causal factor in the development of anxiety.

\section{References}

Baas, Johanna, Jean Milstein, Meghan Donlevy, and Christian Grillon. 2006. "Brainstem Correlates of Defensive States in Humans." Biological Psychiatry 59 (7): 588-93.

Bandettini, Peter A. 2012. "Twenty Years of Functional MRI: The Science and the Stories." NeuroImage 62 (2): 575-88.

Barlow, David H. 2000. "Unraveling the Mysteries of Anxiety and Its Disorders from the Perspective of Emotion Theory." American Psychologist 55 (11): 1247-263. 
Beck, A. T. 1961. "An Inventory for Measuring Depression.” Archives of General Psychiatry 4 (6): 561.

Berens, Anne E., Sarah K. G. Jensen, and Charles A. Nelson. 2017. "Biological Embedding of Childhood Adversity: From Physiological Mechanisms to Clinical Implications." BMC Medicine 15 (1).

Bidet-Caulet, Aurélie, Pierre-Guillaume Barbe, Sylvie Roux, Humsini Viswanath, Catherine Barthélémy, Nicole Bruneau, Robert T. Knight, and Frédérique Bonnet-Brilhault. 2012. "Dynamics of Anticipatory Mechanisms during Predictive Context Processing." European Journal of Neuroscience 36 (7): 2996-3004.

Bishop, Sonia J. 2007. "Neurocognitive Mechanisms of Anxiety: An Integrative Account." Trends in Cognitive Sciences 11 (7): 307-16.

Bzdok, Danilo, Angela R. Laird, Karl Zilles, Peter T. Fox, and Simon B. Eickhoff. 2012. "An Investigation of the Structural, Connectional, and Functional Subspecialization in the Human Amygdala." Human Brain Mapping 34 (12): 3247-266.

Cecchetti, Luca, Ron Kupers, Maurice Ptito, Pietro Pietrini, and Emiliano Ricciardi. 2016. "Are Supramodality and Cross-Modal Plasticity the Yin and Yang of Brain Development? From Blindness to Rehabilitation." Frontiers in Systems Neuroscience 10.

Coan, James A., Hillary S. Schaefer, and Richard J. Davidson. 2006. "Lending a Hand." Psychological Science 17 (12): 1032-039.

Cornwell, Brian R., Johanna M.p. Baas, Linda Johnson, Tom Holroyd, Frederick W. Carver, Shmuel Lissek, and Christian Grillon. 2007. "Neural Responses to Auditory Stimulus Deviance under Threat of Electric Shock Revealed by Spatially-filtered Magnetoencephalography." NeuroImage 37 (1): 282-89.

Cuthbert, Bruce N., Harald T. Schupp, Margaret M. Bradley, Niels Birbaumer, and Peter J. Lang. 2000. "Brain Potentials in Affective Picture Processing: Covariation with Autonomic Arousal and Affective Report." Biological Psychology 52 (2): 95-111. 
Dalton, Kim M., Ned H. Kalin, Thomas M. Grist, and Richard J. Davidson. 2005. "Neural-Cardiac Coupling in Threat-Evoked Anxiety." Journal of Cognitive Neuroscience 17 (6): 969-80.

Dedovic, Katarina, Annie Duchesne, Julie Andrews, Veronika Engert, and Jens C. Pruessner. 2009. "The Brain and the Stress Axis: The Neural Correlates of Cortisol Regulation in Response to Stress." NeuroImage 47 (3): 864-71.

Dieterich, Raoul, Tanja Endrass, and Norbert Kathmann. 2016. “Uncertainty Is Associated with Increased Selective Attention and Sustained Stimulus Processing." Cognitive, Affective, \& Behavioral Neuroscience 16 (3): 447-56.

Dolcos, F. 2006. "Brain Systems Mediating Cognitive Interference by Emotional Distraction.” Journal of Neuroscience 26 (7): 2072-079.

Domínguez-Borràs, Judith, Sebastian Walter Rieger, Corrado CorradiDellacqua, Rémi Neveu, and Patrik Vuilleumier. 2016. "Fear Spreading Across Senses: Visual Emotional Events Alter Cortical Responses to Touch, Audition, and Vision." Cerebral Cortex 27 (1): 68-82.

Driver, Jon, and Toemme Noesselt. 2013. "Multisensory Interplay Reveals Crossmodal Influences on 'Sensory-Specific' Brain Regions, Neural Responses, and Judgments." Neuron 57 (1): 11-23.

Driver, Jon, and Charles Spence. 2000. "Multisensory Perception: Beyond Modularity and Convergence." Current Biology 10 (20).

Duncan, Connie C., Robert J. Barry, John F. Connolly, Catherine Fischer, Patricia T. Michie, Risto Näätänen, John Polich, Ivar Reinvang, and Cyma Van Petten. 2009. "Event-related Potentials in Clinical Research: Guidelines for Eliciting, Recording, and Quantifying Mismatch Negativity, P300, and N400." Clinical Neurophysiology 120 (11): 1883-908.

Dunning, Jonathan P., Sophie Deldonno, and Greg Hajcak. 2013. "The Effects of Contextual Threat and Anxiety on Affective Startle Modulation." Biological Psychology 94 (1): 130-35.

Felmingham, Kim L., Laura F. Stewart, Andrew H. Kemp, and Andrea R. Carr. 2016. "The Impact of High Trait Social Anxiety on Neural Processing of Facial Emotion Expressions in Females.” Biological Psychology 117: 179-86. 
Fernandes, V., and F.l. Osório. 2015. "Are There Associations between Early Emotional Trauma and Anxiety Disorders? Evidence from a Systematic Literature Review and Meta-analysis." European Psychiatry 30 (6): 756-64.

Frassinetti, Francesca, Nadia Bolognini, and Elisabetta Làdavas. 2002. "Enhancement of Visual Perception by Crossmodal Visuo-auditory Interaction." Experimental Brain Research 147 (3): 332-43.

Gilmore, Casey S., Brett A. Clementz, and Patrick Berg. 2009. "Hemispheric Differences in Auditory Oddball Responses during Monaural versus Binaural Stimulation." International Journal of Psychophysiology 73 (3): 326-33.

Godoy, Lívea Dornela, Matheus Teixeira Rossignoli, Polianna Delgino-Pereira, Norberto Garcia-Cairasco and Eduardo Henrique de Lima Umeoka. 2018. "A comprehensive overview on stress neurobiology: basic concepts and clinical implications." Frontiers in Behavioral Neuroscience 12: 127.

Gorka, Stephanie M., Lynne Lieberman, Stewart A. Shankman, and K. Luan Phan. 2017. "Startle Potentiation to Uncertain Threat as a Psychophysiological Indicator of Fear-based Psychopathology: An Examination across Multiple Internalizing Disorders." Journal of Abnormal Psychology 126 (1): 8-18.

Gratton, Gabriele, Michael G.h Coles, and Emanuel Donchin. 1983. "A New Method for Off-line Removal of Ocular Artifact." Electroencephalography and Clinical Neurophysiology 55 (4): 468-84.

Grillon, Christian, Johanna P. Baas, Shmuel Lissek, Kathryn Smith, and Jean Milstein. 2005. "Anxious Responses to Predictable and Unpredictable Aversive Events." Behavioral Neuroscience 118 (5): 916-24.

Grosso, Anna, Giulia Santoni, Eugenio Manassero, Annamaria Renna, and Benedetto Sacchetti. 2018. "A Neuronal Basis for Fear Discrimination in the Lateral Amygdala." Nature Communications 9 (1).

Grupe, Dan W., and Jack B. Nitschke. 2013. "Uncertainty and Anticipation in Anxiety: An Integrated Neurobiological and Psychological Perspective." Nature Reviews Neuroscience 14 (7): 488-501. 
Heimler, Benedetta, Ella Striem-Amit, and Amir Amedi. 2015. "Origins of Task-specific Sensory-independent Organization in the Visual and Auditory Brain: Neuroscience Evidence, Open Questions and Clinical Implications." Current Opinion in Neurobiology 35: 169-77.

Huang, S., Y. Li, W. Zhang, B. Zhang, X. Liu, L. Mo, and Q. Chen. 2015. "Multisensory Competition Is Modulated by Sensory Pathway Interactions with Fronto-Sensorimotor and Default-Mode Network Regions." Journal of Neuroscience 35 (24): 9064-077.

Janak, Patricia H., and Kay M. Tye. 2015. "From Circuits to Behaviour in the Amygdala." Nature 517 (7534): 284-92.

Joëls, Marian, and Tallie Z. Baram. 2009. "The Neuro-symphony of Stress." Nature Reviews Neuroscience 10 (6): 459-66.

Kimble, Matthew, Mariam Boxwala, Whitney Bean, Kristin Maletsky, Jessica Halper, Kaleigh Spollen, and Kevin Fleming. 2014. "The Impact of Hypervigilance: Evidence for a Forward Feedback Loop." Journal of Anxiety Disorders 28 (2): 241-45.

Kloet, E. Ron De, Marian Joëls, and Florian Holsboer. 2005. "Stress and the Brain: From Adaptation to Disease." Nature Reviews Neuroscience 6 (6): 46375.

Krauzlis, Richard J., Amarender R. Bogadhi, James P. Herman, and Anil Bollimunta. 2018. "Selective Attention without a Neocortex." Cortex 102: 16175 .

Lang, Peter J. 2005. "International affective picture system (IAPS): Affective ratings of pictures and instruction manual." Technical report.

Lang, Elmar Wolfgang, Ana Maria Tomé, Ingo Rudolf Keck, Juan Manuel Gorriz and Carlos G. Puntonet. 2012. "Brain connectivity analysis: a short survey." Computational intelligence and neuroscience 8.

Ledoux, Joseph E. 2000. "Emotion Circuits in the Brain." Annual Review of Neuroscience 23 (1): 155-84. 
Lim, S.-L., S. Padmala, and L. Pessoa. 2009. "Segregating the Significant from the Mundane on a Moment-to-moment Basis via Direct and Indirect Amygdala Contributions." Proceedings of the National Academy of Sciences 106 (39): 16841-6846

Lindenberg, Robert, and Lukas Scheef. 2007. "Supramodal Language Comprehension: Role of the Left Temporal Lobe for Listening and Reading." Neuropsychologia 45 (10): 2407-415.

Lissek, Shmuel, Kaebah Orme, Dana J. Mcdowell, Linda L. Johnson, David A. Luckenbaugh, Johanna M. Baas, Brian R. Cornwell, and Christian Grillon. 2007. "Emotion Regulation and Potentiated Startle across Affective Picture and Threat-of-shock Paradigms." Biological Psychology 76 (1-2): 124-33.

Lovelace, Christopher T., Barry E. Stein, and Mark T. Wallace. 2003. "An Irrelevant Light Enhances Auditory Detection in Humans: A Psychophysical Analysis of Multisensory Integration in Stimulus Detection." Cognitive Brain Research 17 2: 447-53.

Macaluso, Emiliano. 2013. "Multisensory Processing in Sensory-Specific Cortical Areas." The Neuroscientist 12 (4): 327-38.

Mcdonald, John J., Wolfgang A. Teder-Sälejärvi, and Steven A. Hillyard. 2000. "Involuntary Orienting to Sound Improves Visual Perception." Nature 407 6806: 906-08.

Milad, Mohammed R., and Gregory J. Quirk. 2012. "Fear Extinction as a Model for Translational Neuroscience: Ten Years of Progress." Annual Review of Psychology 63 (1): 129-51.

Millett, David. 2001. "Hans Berger: From Psychic Energy to the EEG." Perspectives in Biology and Medicine 44 (4): 522-42.

Miller, Earl K., and Jonathan D. Wallis. 2013. "The Prefrontal Cortex and Executive Brain Functions." Fundamental Neuroscience, 1069-089.

Nelson, Brady D., and Greg Hajcak. 2017. "Defensive Motivation and Attention in Anticipation of Different Types of Predictable and Unpredictable Threat: A Startle and Event-related Potential Investigation." Psychophysiology 54 (8): 1180-194. 
Nelson, Brady D., Greg Hajcak, and Stewart A. Shankman. 2015. "Event-related Potentials to Acoustic Startle Probes during the Anticipation of Predictable and Unpredictable Threat." Psychophysiology 52 (7): 887-94.

Niedermeyer, E. 1998. "Frontal Lobe Functions and Dysfunctions." Clinical Electroencephalography 29 (2): 79-90.

Nieuwenhuis, Sander, Gary Aston-Jones, and Jonathan D. Cohen. 2005. "Decision Making, the P3, and the Locus Coeruleus--norepinephrine System." Psychological Bulletin 131 (4): 510-32.

Oostenveld, Robert, and Peter Praamstra. 2001. "The Five Percent Electrode System for High-resolution EEG and ERP Measurements." Clinical Neurophysiology 112 (4): 713-19.

Pezzulo, Giovanni. 2008. "Coordinating with the Future: The Anticipatory Nature of Representation." Minds and Machines 18 (2): 179-225.

Plichta, Michael M., Oliver Grimm, Katrin Morgen, Daniela Mier, Carina Sauer, Leila Haddad, Heike Tost, Christine Esslinger, Peter Kirsch, Adam J. Schwarz, and Andreas Meyer-Lindenberg. 2014. "Amygdala Habituation: A Reliable FMRI Phenotype." NeuroImage 103: 383-90.

Posner, M. 1990. “The Attention System Of The Human Brain.” Annual Review of Neuroscience 13 (1): 25-42.

Posner, Michael I., and Mary K. Rothbart. 2007. "Research on Attention Networks as a Model for the Integration of Psychological Science." Annual Review of Psychology 58 (1): 1-23.

Rapp, Brenda, and Sharma K. Hendel. 2003. "Principles of Cross-modal Competition: Evidence from Deficits of Attention." Psychonomic Bulletin \& Review 10 (1): 210-19.

Rouby, Catherine, Arnaud Fournel, and Moustafa Bensafi. 2016. "The Role of the Senses in Emotion." Emotion Measurement 65-81.

Schmitz, Anja, and Christian Grillon. 2013. "Assessing Fear and Anxiety in Humans Using the Threat of Predictable and Unpredictable Aversive Events (the NPU-threat Test)." Nature Protocols 7 (3): 527-32. 
Schupp, Harald T., J. Stockburger, M. Codispoti, M. Junghofer, A. I. Weike, and A. O. Hamm. 2007. "Selective Visual Attention to Emotion." Journal of Neuroscience 27 (5): 1082-089.

Schupp, Harald T., Bruce N. Cuthbert, Margaret M. Bradley, John T. Cacioppo, Tiffany Ito, and Peter J. Lang. 2000. "Affective Picture Processing: The Late Positive Potential Is Modulated by Motivational Relevance." Psychophysiology 37 (2): 257-61.

Shackman, A. J., J. S. Maxwell, B. W. Mcmenamin, L. L. Greischar, and R. J. Davidson. 2011. "Stress Potentiates Early and Attenuates Late Stages of Visual Processing." Journal of Neuroscience 31 (3): 1156-161.

Sharvit, Gil, Patrik Vuilleumier, Sylvain Delplanque, and Corrado CorradiDell'Acqua. 2015. "Cross-modal and Modality-specific Expectancy Effects between Pain and Disgust." Scientific Reports 5 (1).

Slotnick, Scott D. 2005. Source localization of ERP generators. In Handy, Todd C. Event-related Potentials: A Methods Handbook. Camridge, MA: MIT Press. Spielberger, Charles Donald, and R. L. Gorsuch. 1983. Manual for the Statetrait Anxiety Inventory: Form Y: "Self-evaluation Questionnaire". Palo Alto: Consulting Psychologists Press.

Stein, Barry E., Terrence R. Stanford, and Benjamin A. Rowland. 2009. "The Neural Basis of Multisensory Integration in the Midbrain: Its Organization and Maturation." Hearing Research 258 (1-2): 4-15.

Swartz, Johnna R., Annchen R. Knodt, Spenser R. Radtke, and Ahmad R. Hariri. 2015. "A Neural Biomarker of Psychological Vulnerability to Future Life Stress." Neuron 85 (3): 505-11.

Todd, Rebecca M., and Maria G.m. Manaligod. 2018. "Implicit Guidance of Attention: The Priority State Space Framework." Cortex 102: 121-38.

Vroomen, Jean, and Beatrice De Gelder. 2000. "Sound Enhances Visual Perception: Cross-modal Effects of Auditory Organization on Vision." Journal of Experimental Psychology: Human Perception and Performance 26 (5): 1583590. 
Weinberg, Anna, and Greg Hajcak. 2010. "Beyond Good and Evil: The Timecourse of Neural Activity Elicited by Specific Picture Content." Emotion 10 (6): 767-82.

Wise, Vikki, Alexander C. Mcfarlane, C. Richard Clark, and Malcolm Battersby. 2009. "Event-related Potential and Autonomic Signs of Maladaptive Information Processing during an Auditory Oddball Task in Panic Disorder." International Journal of Psychophysiology 74 (1): 34-44.

Woodman, G. F. 2010. "A Brief Introduction to the Use of Event-related Potentials in Studies of Perception and Attention." Attention, Perception \& Psychophysics 72 (8): 2031-046.

Xiong, Fuxia, and Lubo Zhang. 2013. "Role of the Hypothalamic-pituitaryadrenal Axis in Developmental Programming of Health and Disease." Frontiers in Neuroendocrinology 34 (1): 27-46.

Yang, Jong-Chul, Kwangsung Park, Sung-Jong Eun, Moo-Suk Lee, Jin-Sang Yoon, Il-Seon Shin, Yong-Ku Kim, Tae-Woong Chung, Heoung-Keun Kang, and Gwang-Woo Jeong. 2008. "Assessment of Cerebrocortical Areas Associated with Sexual Arousal in Depressive Women Using Functional MR Imaging." The Journal of Sexual Medicine 5 (3): 602-09.

Zukerman, Gil, Leah Fostick, and Ester Ben-Itzchak. 2018. "Early Automatic Hyperarousal in Response to Neutral Novel Auditory Stimuli among Traumaexposed Individuals with and without PTSD: An ERP Study." Psychophysiology 55 (11). 


\section{Instructions for authors}

The editor welcomes original contributions. By submission of a manuscript an author certifies that the work is original and is not being considered simultaneously by another publisher.

Manuscripts should normally be between 6000-8000 words. All manuscripts will be peer reviewed and only those receiving favourable recommendation will be accepted for publication.

References: please follow Chicago Manual Style, 16th edition: http://www.chicagomanualofstyle.org/home.html. See the instructions on the website www.fuds.si/en/publishing.

Manuscript form: please follow the sample paper on the website www.fuds.si/en/publishing.

Please submit all manuscripts by email to rsc@fuds.si. 